\title{
Intrinsic Sensitivity Limits for Multiparameter Quantum Metrology
}

\author{
Aaron Z. Goldberg $\oplus^{1,2}$ Luis L. Sánchez-Soto ${ }^{3,4}$ and Hugo Ferretti ${ }^{2}$ \\ ${ }^{1}$ National Research Council of Canada, 100 Sussex Drive, Ottawa, Ontario K1A OR6, Canada \\ ${ }^{2}$ Department of Physics, University of Toronto, 60 St. George Street, Toronto, Ontario M5S 1A7, Canada \\ ${ }^{3}$ Departamento de Óptica, Facultad de Física, Universidad Complutense, 28040 Madrid, Spain \\ ${ }^{4}$ Max-Planck-Institute für die Physik des Lichts, 91058 Erlangen, Germany
}

(Received 17 May 2021; revised 30 June 2021; accepted 29 July 2021; published 7 September 2021)

\begin{abstract}
The quantum Cramér-Rao bound is a cornerstone of modern quantum metrology, as it provides the ultimate precision in parameter estimation. In the multiparameter scenario, this bound becomes a matrix inequality, which can be cast to a scalar form with a properly chosen weight matrix. Multiparameter estimation thus elicits trade-offs in the precision with which each parameter can be estimated. We show that, if the information is encoded in a unitary transformation, we can naturally choose the weight matrix as the metric tensor linked to the geometry of the underlying algebra $\mathfrak{i t} \mathfrak{t}(n)$, with applications in numerous fields. This ensures an intrinsic bound that is independent of the choice of parametrization.
\end{abstract}

DOI: 10.1103/PhysRevLett.127.110501

Introduction.-A central challenge in quantum metrology is to develop strategies for enhancing the precision of parameter estimation. The quantum Fisher information (QFI), and the associated quantum Cramér-Rao bound (QCRB), are invaluable tools for this task [1,2], as they characterize the ultimate precision attainable for different classes of probe states. This is crucial to identifying quantum resources that lead to an enhancement in sensitivity versus classical strategies.

It is well established that the maximal sensitivities achievable for the estimation of a single parameter using particle-separable and arbitrary quantum probe states are the shot-noise and Heisenberg limits, respectively [3]. However, much less is known about the corresponding bounds for the simultaneous estimation of multiple parameters. Multiparameter quantum metrology finds many important applications in fields as diverse as imaging [4-6], field sensing [7-9], sensor networks [10-12], and remote sensing [13] to cite but a few examples. In this case, the QCRB is a matrix inequality, and the ultimate bound is generally not saturable for all parameters. This is because the corresponding optimal observables may be incompatible [14-16], and one cannot simultaneously achieve the optimal precision for each individual parameter. Several comprehensive reviews highlight recent progress in this subject area [17-22].

Published by the American Physical Society under the terms of the Creative Commons Attribution 4.0 International license. Further distribution of this work must maintain attribution to the author(s) and the published article's title, journal citation, and DOI. Open access publication funded by the Max Planck Society.
These difficulties have fueled the search for tighter bounds, which prove to be handy for practical implementations. The Holevo Cramér-Rao bound (HCRB) [2] epitomizes the conundrums associated with incompatible observables: its main tenet is to map the matrix QCRB onto a scalar inequality by using a positive-definite weight matrix and then optimize this scalar bound over all physically viable measurement procedures for a given probe state. In this manner, one obtains a weighted mean square error that has to be minimized. This is considered hard to evaluate, even numerically, because it is defined through a constrained minimization over a set of operators. Closed results are known only for very simple models [2325], and the numerical tractability of calculating the HCRB for finite-dimensional systems has been recently considered $[26,27]$.

Surprisingly, little attention has been paid to the properties of the weight matrix: its only role is to give relative weights to the different parameter estimate variances, so different choices lead to different optimal probe states and experimental designs. This entails a significant ambiguity in real-world problems that inherently involve numerous parameters. One idea is to always choose the weight matrix that gives the worst possible total error for an optimal measurement strategy [28]. In this Letter, we discuss whether that matrix can be chosen in a natural way.

To provide a proper answer, we first assume that the parameters are imprinted onto the probe state via a unitary transformation; i.e., we assume that the parameters belong to the group $\mathrm{SU}(n)$. From a geometrical viewpoint, the associated Lie algebra $\mathfrak{g} \mathfrak{t}(n)$ can be endowed with a metric tensor, the Killing-Cartan form, with all the desirable properties [29]. When this metric is used as our weight matrix, we obtain a QCRB with intrinsic properties, 
independent of the choice of parametrization. Such a QCRB can significantly facilitate finding the corresponding optimal states for estimating all of the parameters with these intrinsic weights. We demonstrate the power of this bound for the broad scenario of estimating all of the parameters of an $\mathrm{SU}(n)$ operation, which generalizes known multiparameter estimation results for rotation [9] and multiphase [30] estimation problems. Because of the ubiquity of unitary transformations, this paves the way for solving new problems, such as determining the limits of 3D polarimetry [31-33]; measuring systems of qudits [34-37]; characterizing multiport interferometers [38-41], which have found recent applications in boson sampling [42-44]; and beyond.

Setting the scenario.-We are interested in estimating multiple parameters simultaneously. The typical scheme requires some $d$-dimensional vector of parameters $\boldsymbol{\theta}=\left(\theta_{1}, \ldots, \theta_{d}\right)^{\top} \in \mathbb{R}^{d}$ to be imprinted on a (pure) probe state $|\psi\rangle$, which is shifted by applying a corresponding unitary transformation $U(\boldsymbol{\theta}) \in \mathrm{SU}(n)$ that encodes the parameters $\boldsymbol{\theta}$ (the superscript $T$ denotes the transpose). The output state $\left|\psi_{\boldsymbol{\theta}}\right\rangle=U(\boldsymbol{\theta})|\psi\rangle$ is then detected via a set of measurements, represented by a positive operatorvalued measure (POVM) [1] $\left\{\Pi_{x}\right\}$, where the POVM elements are labeled by an index $x$ that represents the possible outcomes (discrete or continuous) according to Born's rule $p(x \mid \boldsymbol{\theta})=\left\langle\psi_{\boldsymbol{\theta}}\left|\Pi_{x}\right| \psi_{\boldsymbol{\theta}}\right\rangle$.

Often, the protocol is repeated $N$ times using identical copies of the state. From the output results $\mathbf{x}=\left(x_{1}, \ldots, x_{N}\right)^{\top}$, which we assume to be independent and identically distributed, one can construct a joint probability distribution $p(\mathbf{x} \mid \boldsymbol{\theta})=\prod_{j=1}^{N} p\left(x_{j} \mid \boldsymbol{\theta}\right)$ and then infer the vector parameter via an estimator $\hat{\boldsymbol{\theta}}$. In the following, we restrict our attention to locally unbiased estimators, for which $\left\langle\hat{\theta}_{j}\right\rangle=\theta_{j}$ and $d\left\langle\hat{\theta}_{j}\right\rangle / d \theta_{k}=\delta_{j k}$, and we compute average values $\langle\cdot\rangle$ using the probability distribution $p(\mathbf{x} \mid \boldsymbol{\theta})$ [1].

The performance of the estimator can be properly assessed in terms of the covariance matrix $\mathbf{C}_{\psi}(\hat{\boldsymbol{\theta}})$, defined as

$$
\left[\mathbf{C}_{\psi}(\hat{\boldsymbol{\theta}})\right]_{j k}=\left\langle\left(\hat{\theta}_{j}-\theta_{j}\right)\left(\hat{\theta}_{k}-\theta_{k}\right)\right\rangle,
$$

where $j, k=1, \ldots, d$. The diagonal elements are the variances of the different parameters, whereas the nondiagonal elements characterize the possible correlations between various parameters.

To guide the design of real experiments, it is possible to calculate theoretical lower bounds for $\mathbf{C}_{\psi}(\hat{\boldsymbol{\theta}})$. The ultimate limit for any possible POVM is given by the time-honored QCRB, which stipulates that

$$
\mathbf{C}_{\psi}(\hat{\boldsymbol{\theta}}) \succeq \mathbf{Q}_{\psi}^{-1}(\boldsymbol{\theta})
$$

where the matrix inequality $\mathbf{A} \succeq \mathbf{B}$ means that $\mathbf{A}-\mathbf{B}$ is a positive semidefinite matrix. Here, the quantum Fisher information matrix (QFIM) for pure states and unitary evolution takes the particularly simple form [18]

$$
\left[\mathbf{Q}_{\psi}(\boldsymbol{\theta})\right]_{j k}=4 \mathbf{C}_{\psi}\left(H_{j}, H_{k}\right) .
$$

The operators $H_{j}$ are the generators of the transformation, determined through $H_{j}=i U^{\dagger}(\boldsymbol{\theta}) \partial_{\theta_{j}} U(\boldsymbol{\theta})$, and we define the symmetrized covariance between two operators as $\mathbf{C}_{\psi}(A, B)=\frac{1}{2}\langle A B+B A\rangle-\langle A\rangle\langle B\rangle$ and expectation values with respect to $|\psi\rangle$.

How can we optimize a probe state $|\psi\rangle$ given a matrix figure of merit? Starting from a covariance matrix, we can balance the precision of the various parameters by using a weight matrix $\mathbf{W} \succ 0$; this leads to the scalar inequality

${ }_{\mathrm{WMSE}}(\hat{\boldsymbol{\theta}}) \equiv \operatorname{Tr}\left[\mathbf{W} \mathbf{C}_{\psi}(\hat{\boldsymbol{\theta}})\right] \geq \operatorname{Tr}\left[\mathbf{W} \mathbf{Q}_{\psi}^{-1}(\boldsymbol{\theta})\right] \equiv C_{S}(\boldsymbol{\theta})$.

The left-hand side is the so-called weighted mean square error of the estimator wMSE, whereas $C_{S}(\boldsymbol{\theta})$ is a scalar cost function, much in the same spirit as the risk functions used in Bayesian estimation [45]. For a given $\mathbf{W}$, the standard approach is to minimize $C_{S}(\boldsymbol{\theta})$ to derive better parameter estimates. In contradistinction, we address here whether there is an intrinsic choice for the matrix W.

Intrinsic bound.-Let us assume that the unitary process can be represented as $U(\boldsymbol{\theta})=\exp \left[i \boldsymbol{\Omega}_{(\boldsymbol{\theta})} \cdot \mathbf{X}\right]$, where the vector $\boldsymbol{\Omega}_{(\boldsymbol{\theta})}$ encodes the pertinent set of parameters $\boldsymbol{\theta}$ and $\left\{X_{i}\right\}$ are the generators of $\mathrm{SU}(n)$; that is, $\left\{X_{i}\right\}$ comprise a basis of the Lie algebra $\mathfrak{g} \mathfrak{t}(n)$, which consists of traceless Hermitian $n \times n$ complex matrices with $i[\cdot, \cdot]$ as their Lie bracket $[46]([\cdot, \cdot]$ denoting the regular commutator). This form is especially advantageous because it includes all metrological applications in which the parameters are related to either components of a Hamiltonian or an interaction time. For our final results to hold, we require that the vector $\boldsymbol{\Omega}_{(\boldsymbol{\theta})}$ is a smooth injective function of the parameters $\boldsymbol{\theta}$.

A straightforward method for calculating $\partial_{\theta_{j}} U(\boldsymbol{\theta})$ is through Wilcox's formula [47] for a unitary operator $U=\exp (A)$ :

$$
\frac{\partial U}{\partial \lambda}=\int_{0}^{1} \exp [(1-\beta) A] \frac{\partial A}{\partial \lambda} \exp (\beta A) d \beta .
$$

By recalling the definition of the generators, we find

$$
H_{j}=-\frac{\partial \boldsymbol{\Omega}_{\boldsymbol{\theta}}}{\partial \theta_{j}} \cdot \int_{0}^{1} U^{-\beta} \mathbf{X} U^{\beta} d \beta .
$$


Since $U^{-1} \mathbf{X} U \in \mathfrak{g} \mathfrak{t}(n)$, we conclude that $H_{j}$ can be generically expressed as a linear combination of the generators $\left\{X_{i}\right\}$ through

$$
H_{j}=\mathbf{h}_{j} \cdot \mathbf{X},
$$

where the real vectors of coefficients $\mathbf{h}_{j}(\boldsymbol{\theta})$ are computed from Eq. (6) and depend on $\boldsymbol{\Omega}_{\boldsymbol{\theta}}$. The far-reaching result that the generators $H_{j}$ of the transformation are equal to linear combinations of the generators $\left\{X_{i}\right\}$ is essential to finding an intrinsic QCRB that is independent of parametrization. Moreover, this relation will always hold for the same vectors $\mathbf{h}_{j}$ regardless of the particular representation of the group that we use.

In this way, we can immediately work out a compact expression for the QFIM:

$$
\mathbf{Q}_{\psi}(\boldsymbol{\theta})=4 \mathbf{H}^{\top}(\boldsymbol{\theta}) \mathbf{C}_{\psi}(\mathbf{X}) \mathbf{H}(\boldsymbol{\theta})
$$

and the scalar QCRB now reads as

$$
\operatorname{Tr}\left[\mathbf{W} \mathbf{C}_{\psi}(\hat{\boldsymbol{\theta}})\right] \geq \frac{1}{4} \operatorname{Tr}\left[\mathbf{H}^{-1 \top}(\boldsymbol{\theta}) \mathbf{W} \mathbf{H}^{-1}(\boldsymbol{\theta}) \mathbf{C}_{\psi}^{-1}(\mathbf{X})\right] .
$$

The remarkable property of these expressions is that we have separated the parameter dependence $\mathbf{H}(\boldsymbol{\theta})=$ $\left(\mathbf{h}_{1}, \ldots, \mathbf{h}_{d}\right)^{\top}$ from the state dependence that is embodied in $\left[\mathbf{C}_{\psi}(\mathbf{X})\right]_{j k}=\mathbf{C}_{\psi}\left(X_{j}, X_{k}\right)$. This form allows us to easily identify when the QFIM becomes singular, which implies that all $d$ parameters cannot be simultaneously estimated for some probe state and parametrization. For example, when $\mathbf{C}_{\psi}(\mathbf{X})$ is singular, the probe state will never be useful for estimating all $d$ parameters, while, when $\mathbf{H}$ is singular, the coordinate system defined by the parametrization is singular at that specific set of parameters $\boldsymbol{\theta}$ regardless of the probe state. As discussed in Ref. [48], singularities in one coordinate system can be alleviated for specific parameters by switching to a new coordinate system; here, we show how a proper choice of weight matrix $\mathbf{W}$ removes all ambiguities that can arise from the choice of coordinate system.

To proceed, we note that the parameters $\boldsymbol{\theta}$ are coordinates of the group manifold (the parameters $\boldsymbol{\Omega}_{(\boldsymbol{\theta})}$ form an alternative set of coordinates of the manifold), which is compact and simply connected [46]. One can define therein a local metric tensor and through that covariant operations and invariant quantities. As $\mathfrak{g} \mathfrak{i t}(n)$ is compact and semisimple, the Killing form $B(X, Y)=2 n \operatorname{Tr}\left(X^{\dagger} Y\right)$ is nondegenerate and defines a scalar product $(X, Y)=B(X, Y)$ that, in turn, induces a natural metric $d s^{2}=(d U, d U)$, called the Cartan metric. Actually, by expressing the metric as

$$
d s^{2}=\sum_{j k} g_{j k} d \theta_{j} d \theta_{k}
$$

and computing $d U=-i U \sum_{j} H_{j} d \theta_{j}$, we find that the metric explicitly depends on the generators from Eq. (7) through $g_{j k}=\operatorname{Tr}\left(H_{j} H_{k}\right)$. We can then use the orthonormality of the generators $\left(X_{j}, X_{k}\right)=\delta_{i j}$ to determine the components of the metric tensor to be (up to an inessential global constant)

$$
g_{j k}=\mathbf{h}_{j} \cdot \mathbf{h}_{k},
$$

or, in vector form, $\mathbf{g}=\mathbf{H}^{\top} \mathbf{H}$. It follows that, if we choose the Cartan metric as the weight matrix $\mathbf{W}=\mathbf{g}$, all of the parametrization dependence cancels from Eq. (9), and the QCRB becomes

$$
\operatorname{Tr}\left[\mathbf{g C}_{\psi}(\hat{\boldsymbol{\theta}})\right] \geq \frac{1}{4} \operatorname{Tr}\left[\mathbf{C}_{\psi}^{-1}(\mathbf{X})\right]
$$

The only remaining ingredients are the covariances of the generators of $\mathfrak{i n} \mathfrak{u}(n)$ with respect to the original state, or any state along the unitary orbit, as $\operatorname{Tr}\left[\mathbf{C}_{\psi}^{-1}(\mathbf{X})\right]=\operatorname{Tr}\left[\mathbf{C}_{\psi^{\prime}}^{-1}(\mathbf{X})\right]$, with $\left|\psi^{\prime}\right\rangle=U|\psi\rangle$. This is exactly what one requires physically and seems quite elegant. It nicely complements the single-parameter scenario in which the QFI for unitary operations takes the same value anywhere along the unitary orbit, which previously presented peculiar challenges to the estimation of multiple parameters.

Discussion.-To see how our new result works, let us first consider the simplest case of SU(2), which describes rotations, the type of unitary transformation being estimated in standard polarimetry and magnetometry, among other examples. A general element is characterized by the Euler angle parametrization $U(\Phi, \Theta, \Psi)=$ $e^{-i \Phi J_{z}} e^{-i \Theta J_{y}} e^{-i \Psi J_{z}}$, where we have used the standard angular momentum notation $\mathbf{J}$ for the generators. Derivatives with respect to the parameters lead us to

$$
\begin{aligned}
& \mathbf{h}_{\Phi}=(-\sin \Theta \cos \Psi, \sin \Theta \sin \Psi, \cos \Theta)^{\top}, \\
& \mathbf{h}_{\Theta}=(\sin \Psi, \cos \Psi, 0)^{\top}, \\
& \mathbf{h}_{\Psi}=(0,0,1)^{\top},
\end{aligned}
$$

which makes the matrix $\mathbf{H}$ singular when, for example, $\Theta=0$. The metric for this parametrization is

$$
d s^{2}=d \Phi^{2}+2 \cos \Theta d \Phi d \Psi+d \Theta^{2}+d \Psi^{2},
$$

which directly cancels the singularities in $\mathbf{H}$ through $\mathbf{H}^{-1 \top} \mathbf{g H}^{-1}=\mathbb{1}$, as promised.

It is straightforward to show that in this case $\mathbf{C}_{\psi}(\mathbf{J})$ is singular if and only if the probe state is an eigenstate of some angular momentum projection, that is, proportional to $U(\boldsymbol{\theta})|J m\rangle$. States with any definite angular momentum projection cannot be used for simultaneously estimating all three parameters of a rotation. 
To find the most sensitive states we have to minimize $\operatorname{Tr}\left[\mathbf{C}_{\psi}^{-1}(\mathbf{J})\right]$. This is straightforward to optimize because, for any symmetric, positive-semidefinite, invertible matrix $\mathbf{M}$, $\operatorname{Tr}(\mathbb{1})^{2}=\operatorname{Tr}\left(\mathbf{M}^{1 / 2} \mathbf{M}^{-1 / 2}\right)^{2} \leq \operatorname{Tr}(\mathbf{M}) \operatorname{Tr}\left(\mathbf{M}^{-1}\right)$, with equality if and only if $\mathbf{M}$ is proportional to the identity matrix. Since $\operatorname{Tr}\left[\mathbf{C}_{\psi}(\mathbf{J})\right]=J(J+1)-\sum_{k}\left\langle J_{k}\right\rangle^{2}$, we find

$$
\operatorname{Tr}\left[\mathbf{C}_{\psi}^{-1}(\mathbf{J})\right] \geq \frac{9}{J(J+1)},
$$

with the trace of the inverse achieving the minimum only when the state is first-order unpolarized [49] (that is, $\langle\mathbf{J}\rangle=0)$, and when $\mathbf{C}_{\psi}(\mathbf{J}) \propto \mathbb{1}$, as is the case for the socalled Kings of Quantumness [50-52]. We see that having isotropic angular momentum up until second order makes a state most sensitive to arbitrary rotations about arbitrary axes. Our minimum intrinsic QCRB is given by the square of the number of parameters divided by the quadratic Casimir invariant $\mathcal{C}_{2}=J_{x}^{2}+J_{y}^{2}+J_{z}^{2}=J(J+1) \mathbb{1}$.

The conditions guaranteeing the saturation of the QCRB constitute a touchy business [53]. Fortunately, for the case of pure states, a sufficient condition is $\left\langle\left[H_{j}, H_{k}\right]\right\rangle=$ $0, \forall j, k$. These expectation values will vanish for all states with $\langle\mathbf{J}\rangle=0$, so the optimal state will have a saturable QCRB in this case.

These results can be directly extended to the case of a full $\mathrm{SU}(n)$ estimation. A large number of applications across photonics require the capability of implementing arbitrary linear unitary transformations on a set of optical modes. The control and estimation of all of the free parameters of a general modal unitary transformation is thus of paramount importance for many experiments. This includes spatial multiplexing [54,55], neural networks [56], novel imaging techniques [57], quantum circuits [58], and programmable simulators [59]. Sampling the output probability when photons traverse such networks is hard to simulate in a classical computer, and hence it may be a path to achieve quantum supremacy [60].

Using the same trick, we get that $\operatorname{Tr}\left[\mathbf{C}_{\psi}(\mathbf{X})\right]$ is bounded by the quadratic Casimir invariant $\mathcal{C}_{2}=\sum_{j k} g_{j k} X_{j} X_{k} \equiv$ $\tilde{\mathcal{C}}_{2} \rrbracket$ of $\mathrm{SU}(n)$ and, therefore, the second-order unpolarized states

$$
\langle\mathbf{X}\rangle=0, \quad \mathbf{C}_{\psi}(\mathbf{X})=\frac{\mathcal{C}_{2}}{d},
$$

saturate the optimal QCRB for the simultaneous estimation of all $d$ intrinsically weighted parameters. This yields a minimum total variance $\frac{1}{4} d^{2} / \tilde{\mathcal{C}}_{2}$, generalizing Eq. (15). All of the parameters should be simultaneously estimable with Heisenberg-scaling precisions, because the square root of the Casimir invariant $\sqrt{\tilde{\mathcal{C}}_{2}}$, which corresponds to physical quantities such as the number of particles, is quadratic in the labels of the irreducible representation.
Such a protocol outperforms strategies where the parameters are being sequentially estimated with uncorrelated measurements [61-63] by a factor on the order of $d$. Uncorrelated sequential estimation protocols require splitting the physical resources $\mathcal{O}\left(\sqrt{\tilde{\mathcal{C}}_{2}}\right)$ into $d$ parts, then at best estimating each of the $d$ parameters with variance $\mathcal{O}\left[\left(d / \sqrt{\tilde{\mathcal{C}}_{2}}\right)^{2}\right]$, so that the total variance scales as $\mathcal{O}\left(d^{3} / \tilde{\mathcal{C}}_{2}\right)$. This has been seen for the specific examples of multiphase estimation [30], which satisfies $\mathrm{U}(1)^{\otimes n} \subset$ $\mathrm{SU}(n)$ up to a global phase (i.e., modulo the centre of the group), and rotation estimation [9], which satisfies $\mathrm{SU}(2) \subset \mathrm{SU}(n)$. Estimating the components of any subset of $\mathrm{SU}(n)$ can now be similarly optimized using our results.

When estimating the $d=n^{2}-1$ free parameters of $\mathrm{SU}(n)$ transformations among qudits, states satisfying Eq. (16) are again optimal and achieve the Heisenberg limit for simultaneously estimating all $d$ parameters. The relative advantage of such states over Greenberger-HorneZeilinger (GHZ) states [64], which have been deemed as of optimal sensitivity [65], is depicted in Fig. 1 for various values of $n$; GHZ states only achieve shot-noise scaling in this context. This is because GHZ states are optimized for estimating only a subset of the parameters of an $\mathrm{SU}(n)$ transformation, while the intrinsic weight matrix includes all of the parameters.

The tools developed here are expeditious to apply. Consider, for example, SU(3) metrology, which governs physical systems such as symmetric states of $\mathcal{N}$ qutrits. SU(3) transformations are parametrized by eight variables, making the choice of weight matrix $\mathbf{W}$ cumbersome and potentially arbitrary: fixing $\mathbf{W}$ to correspond to

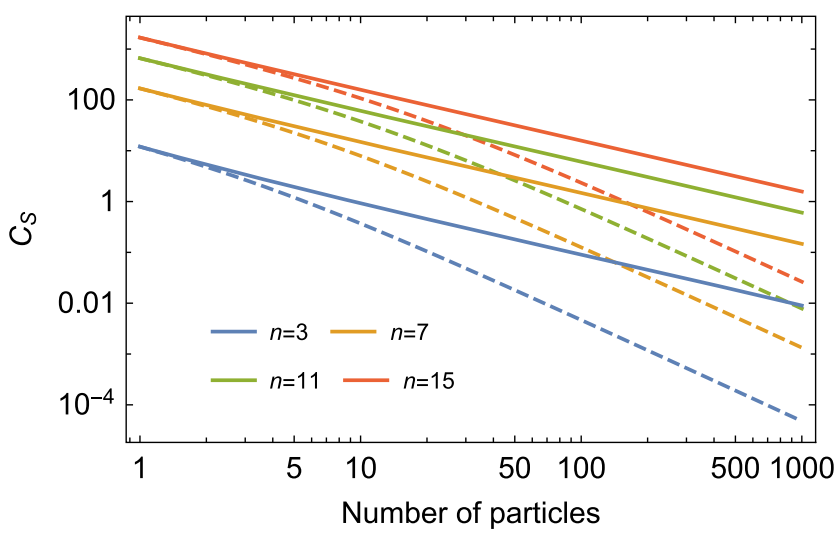

FIG. 1. Minimum total uncertainty $C_{S}$ for estimating all of the parameters of an $\mathrm{SU}(n)$ transformation for our ideal states satisfying Eq. (16) (dashed curves) and GHZ states (solid curves) versus number of particles (photons, qudits, etc.), using our intrinsic weighting of the parameters. While GHZ states, like their two-mode versions known as NOON states, exhibit Heisenberg-scaling precision for estimating some parameters, they convey overall minimum uncertainties exhibiting shot-noise-scaling precision. The ideal states triumph with Heisenberg-scaling precision for the simultaneous estimation of all of the parameters. 
SU(3)'s metric dramatically simplifies the problem, yielding Eq. (12).

One immediate application is to polarimetry: while standard polarimetry estimates the polarization components of a transverse electric field [66], a general beam may have $3 \mathrm{D}$ polarization structure, which requires the knowledge of the 3D Stokes vectors [67-70]. The latter's eight degrees of freedom are exactly the parameters imprinted by $\mathrm{SU}(3)$ operations and constitute the basis of 3D polarimetry, which has plenty of applications in different areas. Our result uniquely simplifies the choice of relative weights for the variances and covariances between all eight parameters.

Equation (16) can readily be used to find ideal states for estimating all eight parameters in three-dimensional polarimetry, without any qualms about how to choose the relative weights of these eight parameters. The former leads to conditions among the coefficients of the states in the photon-number basis, among whose solutions is the set of states

$$
\begin{aligned}
|\psi\rangle= & \frac{1}{\sqrt{3}}(|k-\ell, k, k+\ell\rangle+|k, k+\ell, k-\ell\rangle \\
& +|k+\ell, k-\ell, k\rangle),
\end{aligned}
$$

for pairs of nonzero integers $k=\mathcal{N} / 3$ and $\ell$ satisfying $4 \ell^{2}=3 k(k+1)$. Here, the state $\left|n_{1}, n_{2}, n_{3}\right\rangle$ denotes a Fock state with $n_{1}, n_{2}$, and $n_{3}$ excitations in three respective bosonic modes or a symmetric superposition of $n_{1}, n_{2}$, and $n_{3}$ copies of three respective states. The relative merit of using such states versus GHZ states $(|\mathcal{N}, 0,0\rangle+$ $|0, \mathcal{N}, 0\rangle+|0,0, \mathcal{N}\rangle) / \sqrt{3}$ can be appreciated again in Fig. 1.

Two final comments pertain. First, it is easy to realize that the elegant form (8) for the QFIM also holds for mixed states, where we must employ the replacement [71]

$$
\mathbf{C}_{\psi}(\mathbf{X}) \mapsto \frac{1}{2} \int_{0}^{\infty} d s \operatorname{Tr}\left[(\varrho \mathbf{X}-\mathbf{X} \varrho) e^{-\varrho s}\left(\mathbf{X}^{\top} \varrho-\varrho \mathbf{X}^{\top}\right) e^{-\varrho s}\right],
$$

so the parameter dependencies factor out in terms of $\mathbf{H}(\boldsymbol{\theta})$ and will always disappear in the QCRB if we weight the covariances using the metric. This means that even noisy measurements with imperfect probe states can benefit from our prescription to choose the weight matrix $\mathbf{W}$ to coincide with the metric describing the transformation by using Eq. (12) with the replacement of Eq. (18). Second, the treatment can be repeated much in the same way when the information is encoded either in any of the subgroups of $\mathrm{SU}(n)$ or in channels with symmetries beyond $\mathrm{SU}(n)$. This lets the results take into account scenarios in which not all of the parameters are physically relevant, such as recovering the optimality of NOON states when estimating a single relative phase as a subset of $\mathrm{SU}(2)$.
Concluding remarks. -We have used geometric arguments to show how to obtain an intrinsic QCRB for unitary processes. This directly generalizes many earlier results for multiparameter estimation, notably generalizing the singleparameter estimation result that estimation precision is unchanged along a unitary orbit, and is easily applicable to variegated metrological scenarios. An important advantage of these geometric arguments is that they provide deep connections between quantum estimation and general quantum information tasks.

We would like to thank Hubert de Guise and Pieter Kok for useful discussions. A. Z. G. acknowledges funding from NSERC, the Walter C. Sumner Foundation, and Cray Inc. L.L.S.S. acknowledges financial support from the European Union's Horizon 2020 research and innovation program (Projects ApresSF and Stormytune) and the Spanish Ministerio de Ciencia e Innovación (Grant No. PGC2018- 099183-B-I00). H. F. acknowledges funding from NSERC and CIFAR.

[1] C. W. Helstrom, Quantum Detection and Estimation Theory (Academic, New York, 1976).

[2] A.S. Holevo, Probabilistic and Statistical Aspects of Quantum Theory, 2nd ed. (North Holland, Amsterdam, 2003).

[3] M. G. A. Paris, Quantum estimation for quantum technology, Int. J. Quantum. Inform. 07, 125 (2009).

[4] M. Genovese, Real applications of quantum imaging, J. Opt. 18, 073002 (2016).

[5] S. Z. Ang, R. Nair, and M. Tsang, Quantum limit for twodimensional resolution of two incoherent optical point sources, Phys. Rev. A 95, 063847 (2017).

[6] J. Rehacek, Z. Hradil, B. Stoklasa, M. Paúr, J. Grover, A. Krzic, and L. L. Sánchez-Soto, Multiparameter quantum metrology of incoherent point sources: Towards realistic superresolution, Phys. Rev. A 96, 062107 (2017).

[7] M. Tsang, H. M. Wiseman, and C. M. Caves, Fundamental Quantum Limit to Waveform Estimation, Phys. Rev. Lett. 106, 090401 (2011).

[8] M. Koschorreck, M. Napolitano, B. Dubost, and M. W. Mitchell, High resolution magnetic vector-field imaging with cold atomic ensembles, Appl. Phys. Lett. 98, 074101 (2011).

[9] T. Baumgratz and A. Datta, Quantum Enhanced Estimation of a Multidimensional Field, Phys. Rev. Lett. 116, 030801 (2016).

[10] J. S. Sidhu and P. Kok, Quantum metrology of spatial deformation using arrays of classical and quantum light emitters, Phys. Rev. A 95, 063829 (2017).

[11] J. Nokkala, F. Arzani, F. Galve, R. Zambrini, S. Maniscalco, J. Piilo, N. Treps, and V. Parigi, Reconfigurable optical implementation of quantum complex networks, New J. Phys. 20, 053024 (2018).

[12] J. Rubio, P. A. Knott, T. J. Proctor, and J. A. Dunningham, Quantum sensing networks for the estimation of linear functions, J. Phys. A 53, 344001 (2020). 
[13] V. Ansari, B. Brecht, J. Gil-Lopez, J. M. Donohue, J. Řeháček, Z. Hradil, L. L. Sánchez-Soto, and C. Silberhorn, Achieving the ultimate quantum timing resolution, PRX Quantum 2, 010301 (2021).

[14] H. Zhu, Information complementarity: A new paradigm for decoding quantum incompatibility, Sci. Rep. 5, 14317 (2015).

[15] T. Heinosaari, T. Miyadera, and M. Ziman, An invitation to quantum incompatibility, J. Phys. A 49, 123001 (2016).

[16] S. Ragy, M. Jarzyna, and R. Demkowicz-Dobrzański, Compatibility in multiparameter quantum metrology, Phys. Rev. A 94, 052108 (2016).

[17] M. Szczykulska, T. Baumgratz, and A. Datta, Multiparameter quantum metrology, Adv. Phys. X 1, 621 (2016).

[18] J. S. Sidhu and P. Kok, Geometric perspective on quantum parameter estimation, AVS Quantum Sci. 2, 014701 (2020).

[19] F. Albarelli, M. Barbieri, M. G. Genoni, and I. Gianani, A perspective on multiparameter quantum metrology: From theoretical tools to applications in quantum imaging, Phys. Lett. A 384, 126311 (2020).

[20] E. Polino, M. Valeri, N. Spagnolo, and F. Sciarrino, Photonic quantum metrology, AVS Quantum Sci. 2, 024703 (2020).

[21] R. Demkowicz-Dobrzański, W. Górecki, and M. Guţă, Multi-parameter estimation beyond quantum Fisher information, J. Phys. A 53, 363001 (2020).

[22] A. Z. Goldberg, A. B. Klimov, G. Leuchs, and L. L. Sánchez-Soto, Rotation sensing at the ultimate limit, J. Phys. Photonics 3, 022008 (2021).

[23] J. Suzuki, Explicit formula for the Holevo bound for twoparameter qubit-state estimation problem, J. Math. Phys. (N.Y.) 57, 042201 (2016).

[24] K. Matsumoto, A new approach to the Cramér-Rao-type bound of the pure-state model, J. Phys. A 35, 3111 (2002).

[25] M. Bradshaw, S. M. Assad, and P. K. Lam, A tight CramérRao bound for joint parameter estimation with a pure two-mode squeezed probe, Phys. Lett. A 381, 2598 (2017).

[26] F. Albarelli, J. F. Friel, and A. Datta, Evaluating the Holevo Cramér-Rao Bound for Multiparameter Quantum Metrology, Phys. Rev. Lett. 123, 200503 (2019).

[27] J. S. Sidhu, Y. Ouyang, E. T. Campbell, and P. Kok, Tight Bounds on the Simultaneous Estimation of Incompatible Parameters, Phys. Rev. X 11, 011028 (2021).

[28] F. Belliardo and V. Giovannetti, Incompatibility in quantum parameter estimation, New J. Phys. 23, 063055 (2021).

[29] S. Helgason, Differential Geometry, Lie Groups, and Symmetric Spaces (Academic, New York, 1978).

[30] P. C. Humphreys, M. Barbieri, A. Datta, and I. A. Walmsley, Quantum Enhanced Multiple Phase Estimation, Phys. Rev. Lett. 111, 070403 (2013).

[31] J. Ellis and A. Dogariu, Optical Polarimetry of Random Fields, Phys. Rev. Lett. 95, 203905 (2005).

[32] J. J. Gil, Interpretation of the coherency matrix for threedimensional polarization states, Phys. Rev. A 90, 043858 (2014).

[33] J. J. Gil, A. T. Friberg, T. Setälä, and I. San José, Structure of polarimetric purity of three-dimensional polarization states, Phys. Rev. A 95, 053856 (2017).
[34] R. T. Thew, K. Nemoto, A. G. White, and W. J. Munro, Qudit quantum-state tomography, Phys. Rev. A 66, 012303 (2002).

[35] G. Molina-Terriza, A. Vaziri, J. Řeháček, Z. Hradil, and A. Zeilinger, Triggered Qutrits for Quantum Communication Protocols, Phys. Rev. Lett. 92, 167903 (2004).

[36] G. Lima, L. Neves, R. Guzmán, E. S. Gómez, W. A. T. Nogueira, A. Delgado, A. Vargas, and C. Saavedra, Experimental quantum tomography of photonic qudits via mutually unbiased basis, Opt. Express 19, 3542 (2011).

[37] M. Rambach, M. Qaryan, M. Kewming, C. Ferrie, A. G. White, and J. Romero, Robust and Efficient High-Dimensional Quantum State Tomography, Phys. Rev. Lett. 126, 100402 (2021).

[38] M. Reck, A. Zeilinger, H. J. Bernstein, and P. Bertani, Experimental Realization of Any Discrete Unitary Operator, Phys. Rev. Lett. 73, 58 (1994).

[39] S.-H. Tan, Y. Y. Gao, H. de Guise, and B. C. Sanders, SU(3) Quantum Interferometry with Single-Photon Input Pulses, Phys. Rev. Lett. 110, 113603 (2013).

[40] W. R. Clements, P. C. Humphreys, B. J. Metcalf, W. S. Kolthammer, and I. A. Walmsley, Optimal design for universal multiport interferometers, Optica 3, 1460 (2016).

[41] N. Harris, D. Bunandar, M. Pant, G. R. Steinbrecher, J. Mower, M. Prabhu, T. Baehr-Jones, M. Hochberg, and D. Englund, Large-scale quantum photonic circuits in silicon, Nanophotonics 5, 456 (2016).

[42] S. Aaronson and A. Arkhipov, The computational complexity of linear optics, in Proceedings of the 43rd Annual ACM Symposium on Theory of Computing, STOC' 11 (New York, NY, USA, 2011), pp. 333-342.

[43] M. Tillmann, B. Dakić, R. Heilmann, S. Nolte, A. Szameit, and P. Walther, Experimental boson sampling, Nat. Photonics 7, 540 (2013).

[44] A. Crespi, R. Osellame, R. Ramponi, D. J. Brod, E. F. Galvao, N. Spagnolo, C. Vitelli, P. Maiorino, E.and Mataloni, and F. Sciarrino, Integrated multimode interferometers with arbitrary designs for photonic boson sampling, Nat. Photonics 7, 545 (2013).

[45] S. M. Kay, Fundamentals of Statistical Signal Processing (Prentice Hall, Upper Saddle River, 1993), Vol. 1.

[46] J.F. Cornwell, Group Theory in Physics (Academic, New York, 1984), Vol. II.

[47] R. M. Wilcox, Exponential operators and parameter differentiation in quantum physics, J. Math. Phys. (N.Y.) 8, 962 (1967).

[48] A. Z. Goldberg and D. F. V. James, Quantum-limited Euler angle measurements using anticoherent states, Phys. Rev. A 98, 032113 (2018).

[49] A. Z. Goldberg, P. de la Hoz, G. Björk, A. B. Klimov, M. Grassl, G. Leuchs, and L. L. Sánchez-Soto, Quantum concepts in optical polarization, Adv. Opt. Photonics 13, 1 (2021).

[50] G. Björk, A. B. Klimov, P. de la Hoz, M. Grassl, G. Leuchs, and L. L. Sánchez-Soto, Extremal quantum states and their Majorana constellations, Phys. Rev. A 92, 031801(R) (2015).

[51] F. Bouchard, P. de la Hoz, G. Björk, R. W. Boyd, M. Grassl, Z. Hradil, E. Karimi, A. B. Klimov, G. Leuchs, J. Řeháček, and L. L. Sánchez-Soto, Quantum metrology at the limit 
with extremal Majorana constellations, Optica 4, 1429 (2017).

[52] A. Z. Goldberg, A. B. Klimov, M. Grassl, G. Leuchs, and L. L. Sánchez-Soto, Extremal quantum states, AVS Quantum Sci. 2, 044701 (2020).

[53] A. Fujiwara, Multi-parameter pure state estimation based on the right logarithmic derivative, Tokyo University, Math. Eng. Technical Report No. 94-9, 1994.

[54] J.-F. Morizur, L. Nicholls, P. Jian, S. Armstrong, N. Treps, B. Hage, M. Hsu, W. Bowen, J. Janousek, and H.-A. Bachor, Programmable unitary spatial mode manipulation, J. Opt. Soc. Am. A 27, 2524 (2010).

[55] N. Bozinovic, Y. Yue, Y. Ren, M. Tur, P. Kristensen, H. Huang, A. E. Willner, and S. Ramachandran, Terabit-scale orbital angular momentum mode division multiplexing in fibers, Science 340, 1545 (2013).

[56] Y. Shen, N. C. Harris, S. Skirlo, M. Prabhu, T. Baehr-Jones, M. Hochberg, X. Sun, S. Zhao, H. Larochelle, D. Englund, and M. Soljačić, Deep learning with coherent nanophotonic circuits, Nat. Photonics 11, 441 (2017).

[57] S. M. Popoff, G. Lerosey, R. Carminati, M. Fink, A. C. Boccara, and S. Gigan, Measuring the Transmission Matrix in Optics: An Approach to the Study and Control of Light Propagation in Disordered Media, Phys. Rev. Lett. 104, 100601 (2010).

[58] A. Politi, M. J. Cryan, J. G. Rarity, S. Yu, and J. L. O'Brien, Silica-on-silicon waveguide quantum circuits, Science 320, 646 (2008).

[59] N. C. Harris, G. R. Steinbrecher, M. Prabhu, Y. Lahini, J. Mower, D. Bunandar, C. Chen, F. N. C. Wong, T. Baehr-Jones, M. Hochberg, S. Lloyd, and D. Englund, Quantum transport simulations in a programmable nanophotonic processor, Nat. Photonics 11, 447 (2017).
[60] H.-S. Zhong et al., Quantum computational advantage using photons, Science 370, 1460 (2020).

[61] V. Giovannetti, S. Lloyd, and L. Maccone, Quantum Metrology, Phys. Rev. Lett. 96, 010401 (2006).

[62] V. Giovannetti, S. Lloyd, and L. Maccone, Advances in quantum metrology, Nat. Photonics 5, 222 (2011).

[63] H. Yuan, Sequential Feedback Scheme Outperforms the Parallel Scheme for Hamiltonian Parameter Estimation, Phys. Rev. Lett. 117, 160801 (2016).

[64] D. M. Greenberger, M. A. Horne, A. Shimony, and A. Zeilinger, Bell's theorem without inequalities, Am. J. Phys. 58, 1131 (1990).

[65] V. Giovannetti, S. Lloyd, and L. Maccone, Quantumenhanced measurements: Beating the standard quantum limit, Science 306, 1330 (2004).

[66] A.Z. Goldberg, Quantum theory of polarimetry: From quantum operations to Mueller matrices, Phys. Rev. Research 2, 023038 (2020).

[67] M. R. Dennis, Geometric interpretation of the threedimensional coherence matrix for nonparaxial polarization, J. Opt. A 6, S26 (2004).

[68] A. Luis, Polarization distribution and degree of polarization for three-dimensional quantum light fields, Phys. Rev. A 71, 063815 (2005).

[69] J. J. Gil and I. San José, 3D polarimetric purity, Opt. Commun. 283, 4430 (2010).

[70] C. J. R. Sheppard, Geometric representation for partial polarization in three dimensions, Opt. Lett. 37, 2772 (2012).

[71] D. Petz and C. Ghinea, Introduction to quantum Fisher information, in Quantum Probability and Related Topics (World Scientific, Singapore, 2011), Vol. 27, pp. 261-281. 